\title{
Editorial
}

\section{ICT Innovations in Future Smart Cars}

\author{
Chyi-Ren Dow, ${ }^{1}$ Cheng-Min Lin, ${ }^{2}$ Waleed W. Smari, ${ }^{3}$ \\ Chien-Chung Wu, ${ }^{4}$ and Kuo-Kun Tseng ${ }^{5}$
}

${ }^{1}$ Department of Information Engineering and Computer Science, Feng Chia University, No. 100, Wenhwa Road, Seatwen, Taichung 40724, Taiwan

${ }^{2}$ Department of Digital Living Innovation, Nan Kai University of Technology, No. 568, Chung Cheng Road, Tsao Tun 54243, Nantou, Taiwan

${ }^{3}$ Ball Aerospace \& Technologies Corp., Fairborn, OH 45324, USA

${ }^{4}$ Department of Computer Science and Information Engineering, Southern Taiwan University, No. 1, Nan-Tai Street, Yung-Kang 71005, Tainan, Taiwan

${ }^{5}$ Harbin Institute of Technology Shenzhen Graduate School, D424, HIT Campus of Shenzhen, University Town, Xili, Nanshan, Shenzhen, Guangdong, China

Correspondence should be addressed to Chyi-Ren Dow; crdow@fcu.edu.tw

Received 23 April 2013; Accepted 23 April 2013

Copyright (C) 2013 Chyi-Ren Dow et al. This is an open access article distributed under the Creative Commons Attribution License, which permits unrestricted use, distribution, and reproduction in any medium, provided the original work is properly cited.

This special issue focuses on innovations of information communication technology (ICT) in future smart cars. ICT is a key technology for developing smart cars, its usage in current vehicles is steadily progressing, and the related research issues are very popular and challenging, especially for future smart cars. The objective of this special issue is to provide a leading forum for timely, in-depth presentation of recent advances in services or innovational technologies for future smart cars.

Six papers, ranging in a spectrum from basic theoretical research to solid application research, are included in this special issue. In "Performance evaluation of IEEE 1609 WAVE for vehicular communications," the authors proposed a Markov chain model to support IEEE 802.11 EDCA with transmit opportunity (TXOP) mechanism and presented a more accurate analysis under nonideal channel environment. In "Palm personal identification for vehicular security with a mobile device," the focus is placed on a palm image recognition method to identify individual for vehicular application. In "Detection of overhead contact lines with a 2Ddigital-beamforming radar system for automatic guidance of trolley trucks," a radar system with two-dimensional digital beamforming capability is proposed to offer a compact measurement solution. In "Efficient noninteractive secure protocol enforcing privacy in vehicle-to-roadside communication networks," the authors proposed an efficient noninteractive secure protocol to preserve privacy of drivers in vehicle-toroadside (V2R) communication. In "A driver face monitoring system for fatigue and distraction detection," a novel approach is introduced for driver hypovigilance detection based on the symptoms related to face and eye region. In "A geo-aware and VRP-based public bicycle redistribution system," an actual path distance optimization method is proposed for VRP to adapt the several additional constraints of road problems.

\section{Acknowledgments}

We would like to thank all the authors of this special issue for their contributions. We are also grateful to all the reviewers for their high quality and timely expert reviews that provided many valuable suggestions to the authors. We hope that you enjoy the special issue and find the articles informative and useful.

Chyi-Ren Dow Cheng-Min Lin Waleed W. Smari Chien-Chung Wu Kuo-Kun Tseng 

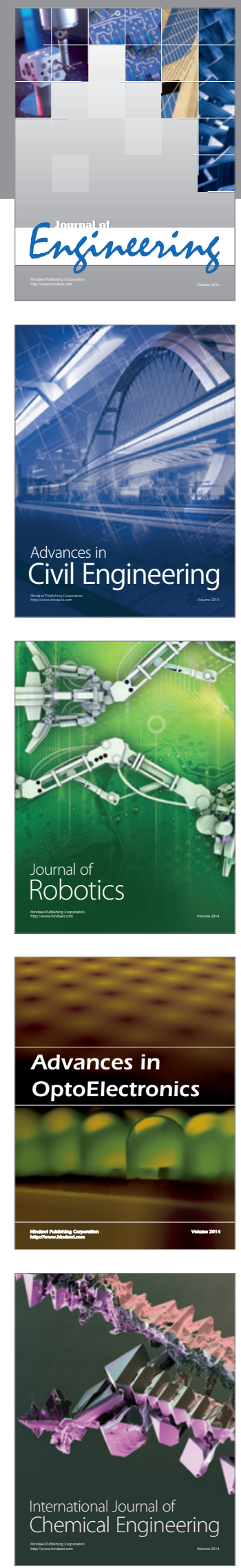

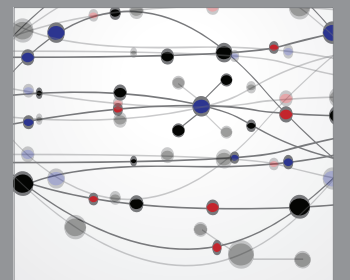

The Scientific World Journal
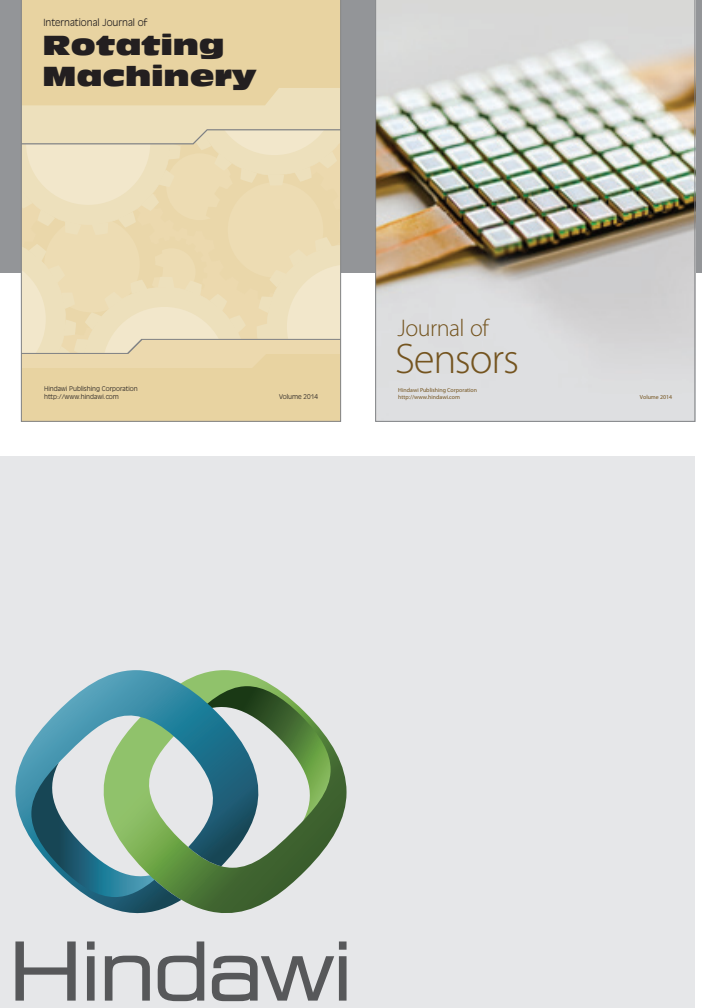

Submit your manuscripts at http://www.hindawi.com
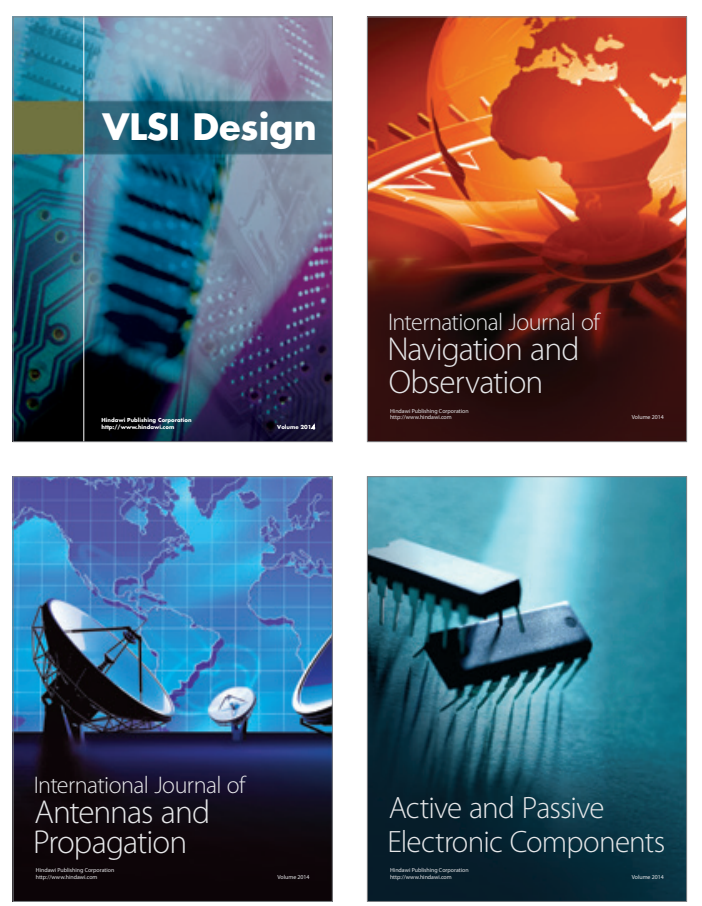
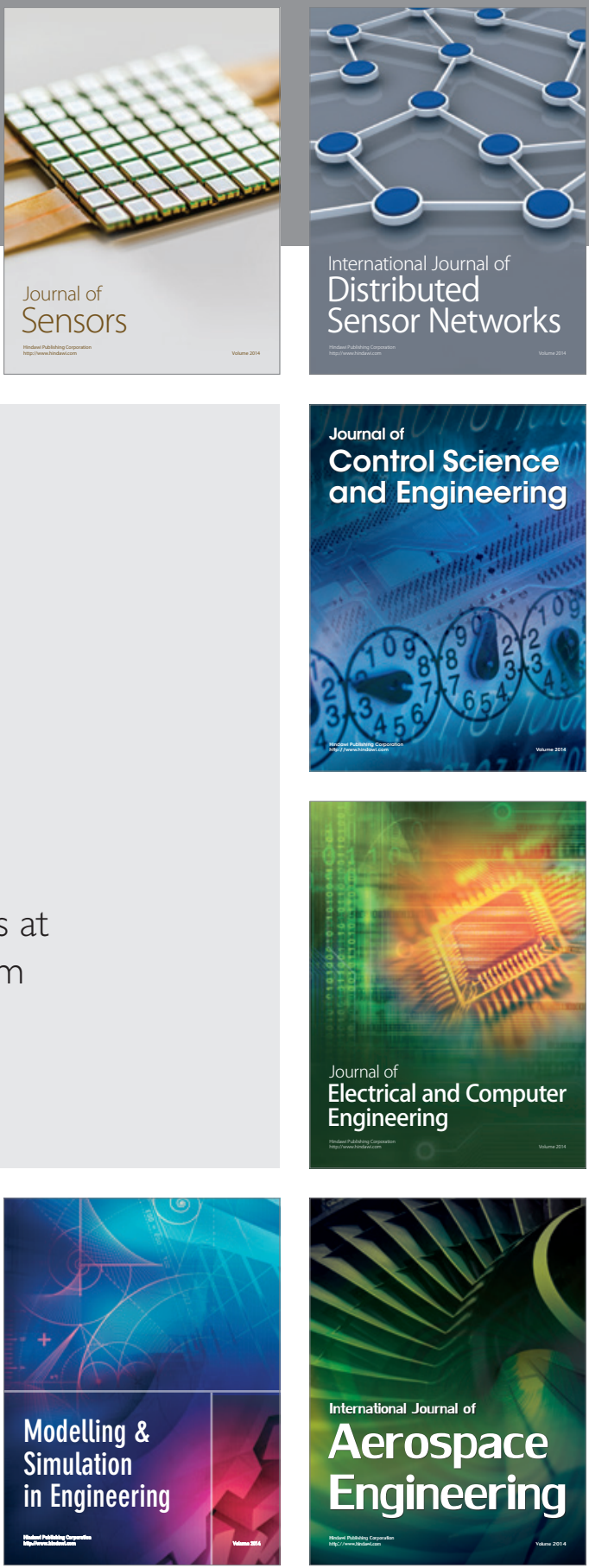

Journal of

Control Science

and Engineering
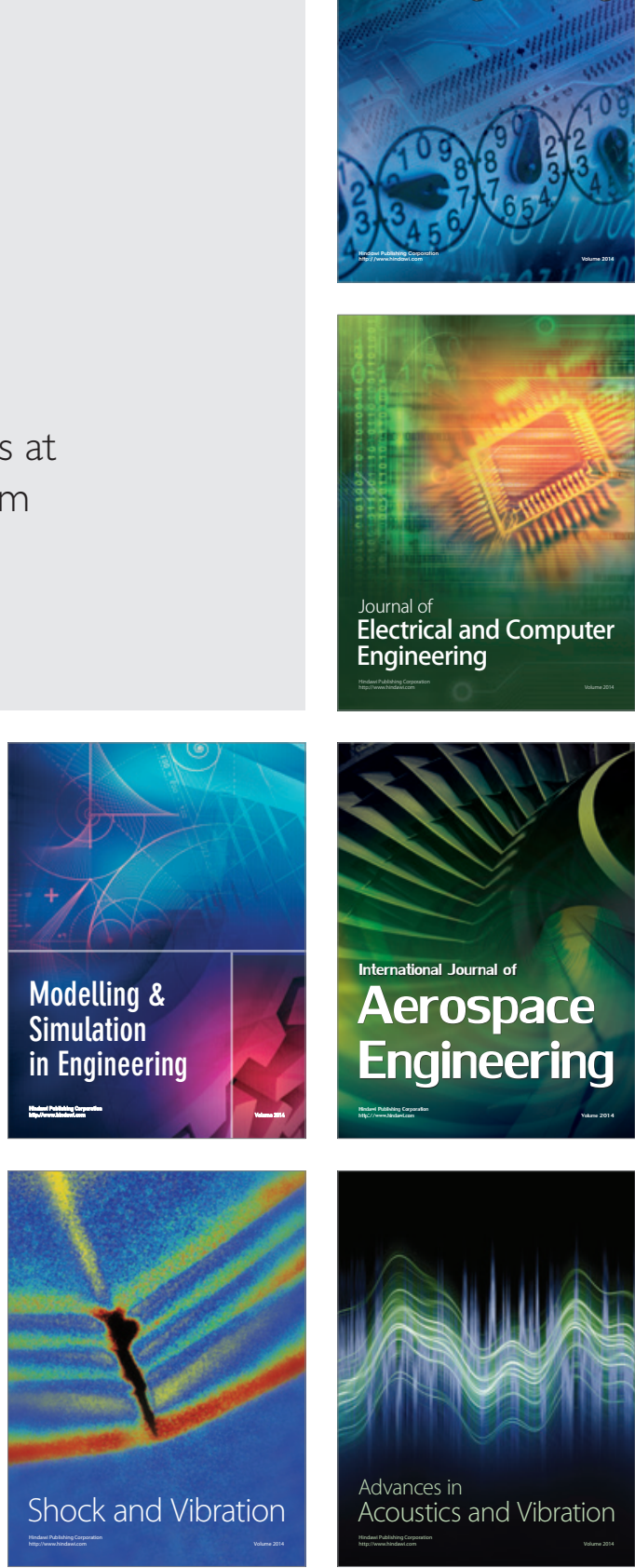